International Journal Of Microwave Engineering (JMICRO) Vol.1, No.3, July 2016

\title{
A DESIGN AND ANALYSIS OF COMPACT MICROSTRIP BANDPASS FILTER WITH INTEGRATED LNA FOR 0.8 TO $2.7 \mathrm{GHZ}$
}

\author{
Mayur B. Chavan ${ }^{1}$ and Prof. Rohita P. Patil ${ }^{2}$ \\ PG Student (VLSI \& Embedded) Skncoe, Vadgoan Pune, India ${ }^{1}$ \\ Assistant Professor, E\&TC Department, Skncoe Vadgoan, Pune, India ${ }^{2}$
}

\begin{abstract}
This paper represents the design and analysis of the Microstrip wideband integrated low noise amplifier Bandpass filter for the frequency range of $0.8 \mathrm{GHz}$ to $2.7 \mathrm{GHz}$. This frequency range is chosen for the design as most of the wireless applications work in this frequency range. The paper uses the compact design structure for designing the wideband filter from ref [5]. The filter has good performance in pass band as well as in stopband of the filter. The filter has the compact size and higher selectivity of 0.923 and the input return loss below $10 \mathrm{~dB}$ and the output return loss less than $10 \mathrm{~dB}$ for the whole frequency range. The integrated low noise amplifier is from the analog devices ADL5544 which has the gain of $15 \mathrm{~dB}$ at $0.8 \mathrm{GHz}$ and the gain roll of $2 \mathrm{~dB}$ in the whole frequency band of the filter. The filter hardware is fabricated and tested with the network analyzer from Rohde \& Schwarz model no ZVH8 which can measure from 100 $\mathrm{KHz}$ to $8 \mathrm{GHz}$. The simulated and the measured results are in good agreement with each other.
\end{abstract}

\section{KEYWORDS}

Bandpass filter (BPF), multiple-mode resonator (MMR), stepped impedance stub load resonator (SISLR), ultra wideband (UWB), Vector network Analyzer (VNA).

\section{INTRODUCTION}

In 2002 the Federal communication Commission(FCC) set the frequency range of the ultrawideband (UWB) as the frequency from $3.1 \mathrm{GHz}$ to $10.6 \mathrm{GHz}$. Since then the advancement in the development of various filters and couplers designed to work in this range started taking growth. Various designs of filters were designed like ring filters multi- mode resonators as shown in ref[1], [2], [3], [4]. The requirement slowly started shifting to the compactness with the good performance in the pass band as well in stopband. The various disadvantages of the Microstrip were considered like harmonic distortion Microstrip losses at higher frequencies etc. Later the multi-mode resonators were used which were able to give good performance in passband but smaller stopband ref [1], [2]. Various other techniques like defective ground were introduced later to have a wider stopbandref [6]. The actual motivation to design the band pass filter for any application is to save the time and the money required for the designing and fabricating the various filters for different applications which are running on different frequencies. By designing the Bandpass filter we actually cuts off the need to design various narrowband filter and use the same Bandpass filter for every application even if the application is running on different frequency. Just that the frequency of operation of the application should be in the passband of the Bandpass filter. Adding to this the gain of the Bandpass filter should be as maximum as possible 
also the input and output return loss of the filter should show some acceptable figures. Dealing with the performance of the filter we cannot ignore the other factors such as size and compactness of the design. As the advancement of technology is taking a mass growth the size will be the only major factor than others. Taking this into account the filter with compact structure is chosen for the frequency which has the maximum wireless applications.

In ref [5] the size of the filter was reduced improving the performance of the filter in the passband as well as in stopband.The size of the filter is reduced as compared to the filter designed in ref [4]. The Fig. 1 shows that the filter uses only a single SIS connected at the center of the uniform impedance transmission line and an aperture-backed beneath three inter-digital parallel coupled lines at connected at each side of the filter for coupling enhancement. The adopted method leads to a simplified objective function with a minimum number of variables to avoid convergence and implementation problems.

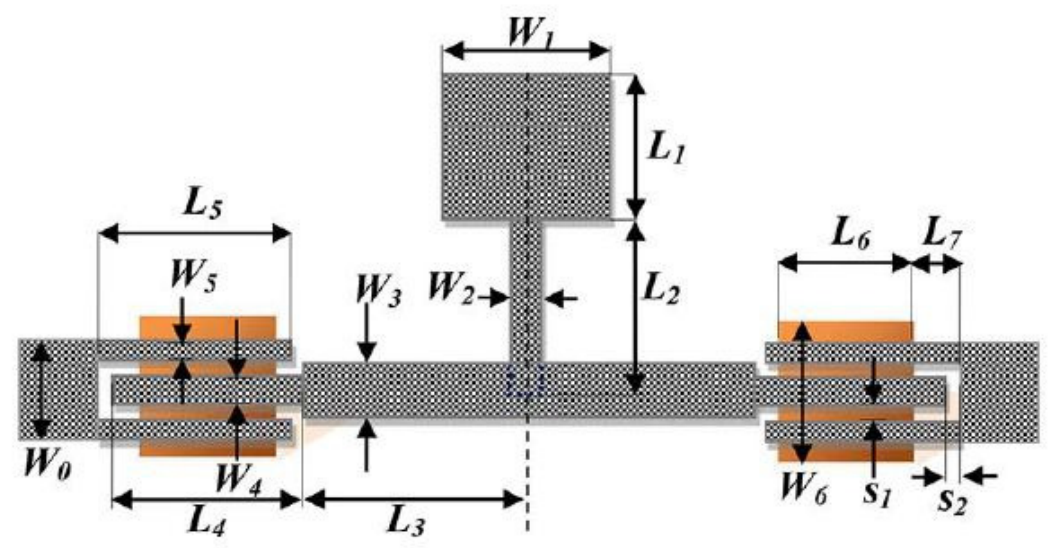

Fig.1 UWB Bandpass filter in ref [5]

In ref [4], the filter size miniaturization was the major challenge faced by the design engineers so this paper proposed a Novel UWB Bandpass filter using stub load multiple mode resonator as shown in Fig.2.

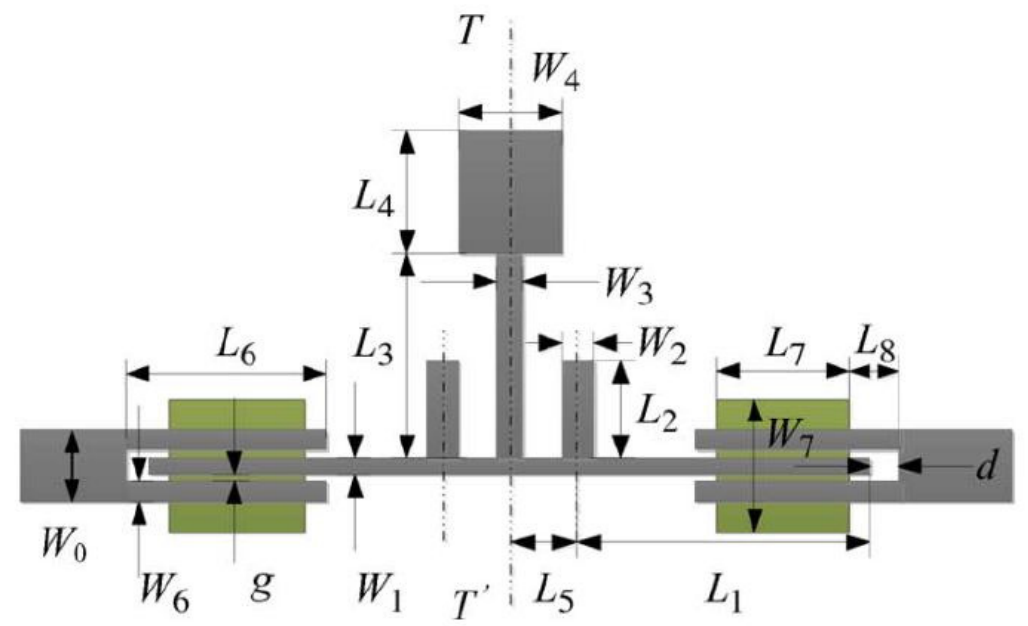

Fig.2 Structure of the SISLR in ref [4] 
This filter had the filter size less as compared to the filter proposed in ref [3]. As shown in Fig.2 the filter used a uniform impedance resonator and consisted of the SIS (Stepped Impedance Stub) at the center and two extra added open stubs at the side of the center stub placed symmetrically around the center. The MMR consists of three open stubs in a uniform impedance resonator, and five modes, including two odd modes and three even modes within the desired band are combined to realize UWB passband.

In ref [3], the novel stepped impedance stub loaded resonator (SISLR) was proposed to design UWB BPF. The previously designed SIR type UWB BPF as shown in Fig.5 showed good performance in passband except the Stopbands suffer the slow increase in attenuation and there were no longer enough degree of freedom for effective control of resonant frequencies.

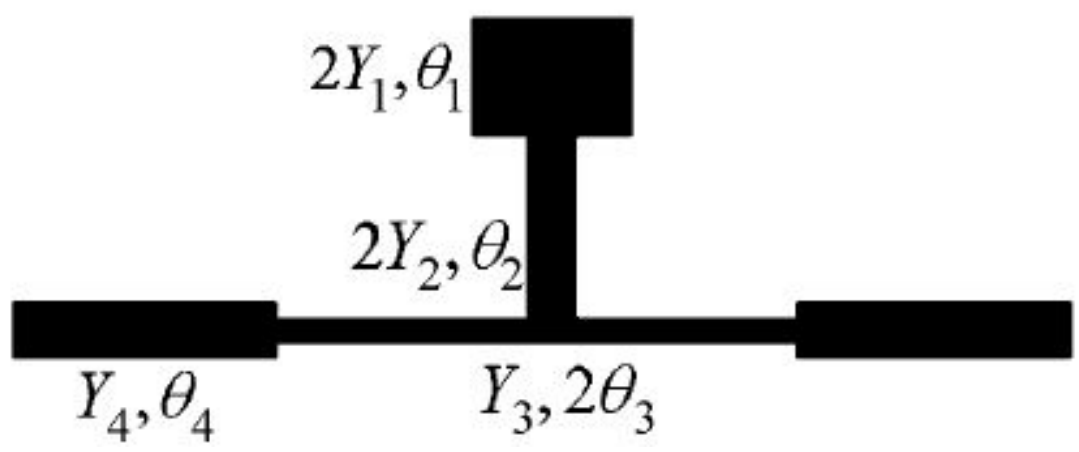

Fig.3 Basic structure of SISLR in ref [3]

This resonator as shown in Fig. 3 has more degrees of adjusting freedom to control its resonant frequencies, which results in conveniently relocating the required resonant modes within the UWB band.The basic structure of the proposed SISLR is shown in Fig.3. It consists of a traditional SIR with the characteristic admittance, and electrical lengths and, which is tappedconnected to a stepped-impedance stub (SIS) in the center. The SIS is also made of transmissionline sections of characteristic admittance, and electrical length. Since the SISLR is symmetrical in structure, odd- and even-mode analysis can be adopted to characterize it.

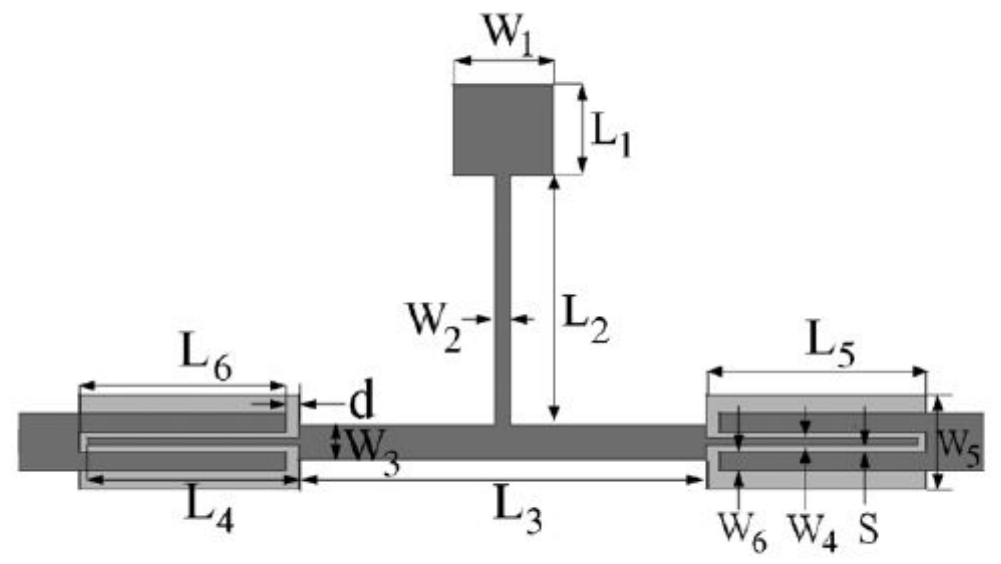

Fig.4 Configuration of the UWB SISLR in [3] 
In ref [2] the Microstrip line stepped impedance stub loaded MMR was proposed in Fig.5.As discussed in ref [1], the first three resonant modes in the stepped-impedance MMR can be quasiequally allocated within the concerned UWB passband by adjusting width/length ratios of centralto-side sections. However, this MMR-based filter usually suffers from a high insertion loss of about $2.0 \mathrm{~dB}$ in the upper UWB passband and a narrow upper stopband of 11.0 to $14.0 \mathrm{GHz}$. The former is mainly caused by parasitic radiation from the central part with wide strip conductor at high frequencies, while the latter is due to the 4th resonant mode in this stepped-impedance MMR.As shown in Fig.5, the proposed stub-loaded MMR is formed by properly attaching one single open-ended stub in the middle and two identical ones in the two symmetrical positions. The lengths of the central stub and side stubs are indicated by Lc and Ls, respectively. In this way, the first four resonant modes expect to be relocated within the UWB passband while pushing up the fifth mode to make up a wide upper stopband.

Compared with the conventional multi-mode resonator in ref [1], this filter design had an extra stepped-impedance stub loaded in the center. The performance of the filter was good but was large in size.

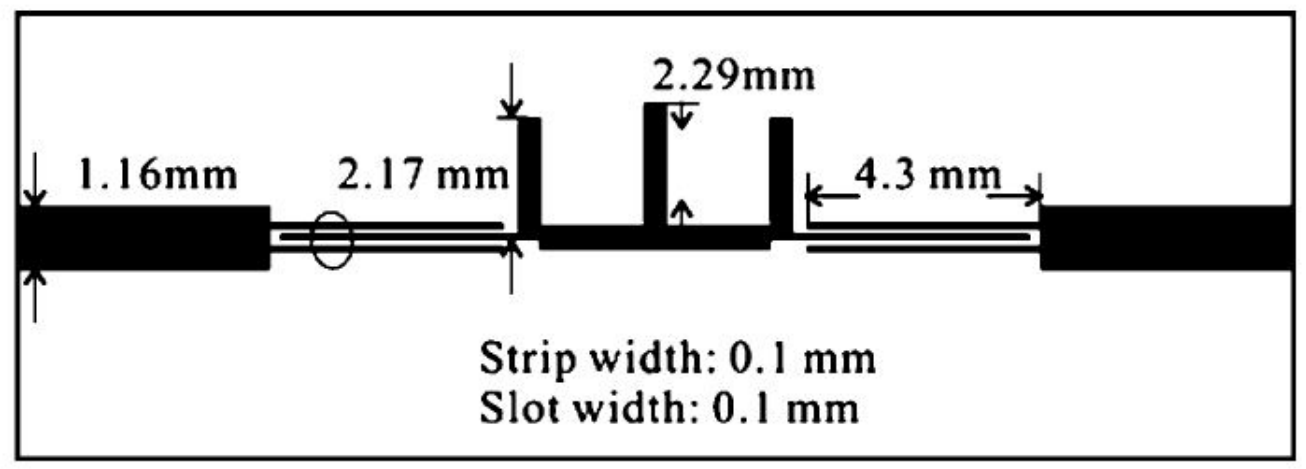

Fig.5 Configuration of the proposed UWB BPF based on stub-loaded MMR in ref [2]

In ref [1], the initially proposed UWB Bandpass filter using a Microstrip line multiple-mode resonator (MMR) was presented as shown in Fig.6. Here the MMR has been properly modified in configuration so as to reallocate its first three resonant modes close to lower-end, center, and upper-end of the targeted UWB passband. Also, the coupling degree of the input/output parallelcoupled line sections is largely raised.

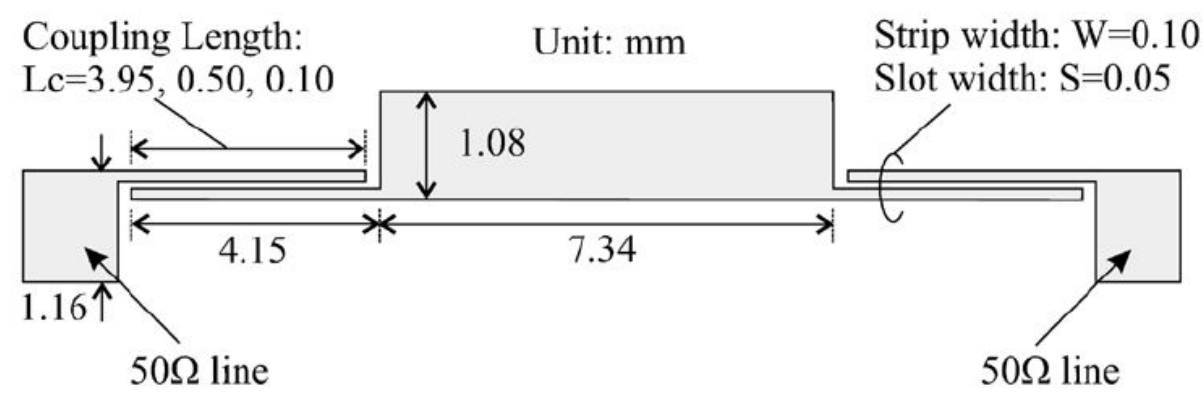

Fig.6 Schematic of the compact Microstrip-line UWB Bandpass filter ref [1] 
At the central frequency of the UWB passband, i.e., $6.85 \mathrm{GHz}$, the MMR consists of one halfwavelength low-impedance line section in the center and two identical $\lambda / 4$ high-impedance line sections at the two sides. With respect to the configuration, the proposed MMR was categorized as a so-called stepped-impedance resonator (SIR). As a non-uniform transmission line resonator, the SIR was proposed in to enlarge the frequency spacing between the first and second-order resonant modes so as to effectively widen the upper stopband above the dominant passband of a Bandpass filter. Here, all the first three resonant modes are taken into account together and they are applied to make up a wide dominant passband. In this case, the first and third-order resonant frequencies basically determine the lower and upper cutoff frequencies of a wide passband. Further the two additional transmission poles in the $\lambda / 4$ parallel-coupled lines, a UWB filter can be built up with good insertion and return loss in the entire passband of concern.

\section{ANALYSIS AND DESIGN OF FILTER}

The filter consists of a uniform transmission line of impedance $\mathrm{Z}$ and has and electrical length of $2 \theta_{C}$ and the characteristic admittance $Y_{C}$. In the structure of the filter as shown in Fig.7the stepped impedance step is connected at the center of the uniform transmission line which has the characteristic impedance of $Z_{A}$ and $Z_{B}$ with the characteristic admittance as $Y_{A}$ and $Y_{B}$ with the electrical length of $\theta_{A}$ and $\theta_{B}$. The actual performance of the ideal filter should be that the gain of the filter should be $S_{21}=1$ for the full passband of interest and then should have sharp cutoff after the pass band. This actually means that filter which is fed by the input impedance of 50 ohms is matched with the impedance of filter for the whole frequency band, where the input impedance of $Z_{\text {in }}=50 \mathrm{ohms}$ and the output impedance of $Z_{\text {out }}=50 \mathrm{ohms}$. Now this condition can be well expressed through the given objective function.

$$
F_{\text {OBJECTIVE }}=\sum_{f_{\text {min }}}^{f_{\text {max }}}\left(\left|\operatorname{Real}\left(Z_{\text {in }}-50\right)\right|+\left|\operatorname{imag}\left(Z_{\text {in }}\right)\right|\right)<\varepsilon
$$

Where $F_{N \max }=F_{\max } / F_{0}$ and $F_{N \min }=F_{\min } / F_{0}$. For the frequency of concern this objective function should be less than the convergence tolerance $\varepsilon$. The value of the convergence tolerance is taken as $\varepsilon=10^{-4}$. The objective function mentioned above is very complicated as it consists of the real and the imaginary parts of the impedance of the filter. Simplification of the above function is required to solve the equation so the filter is divided in to two parts ref [5]. This can be shown in the Fig.8.

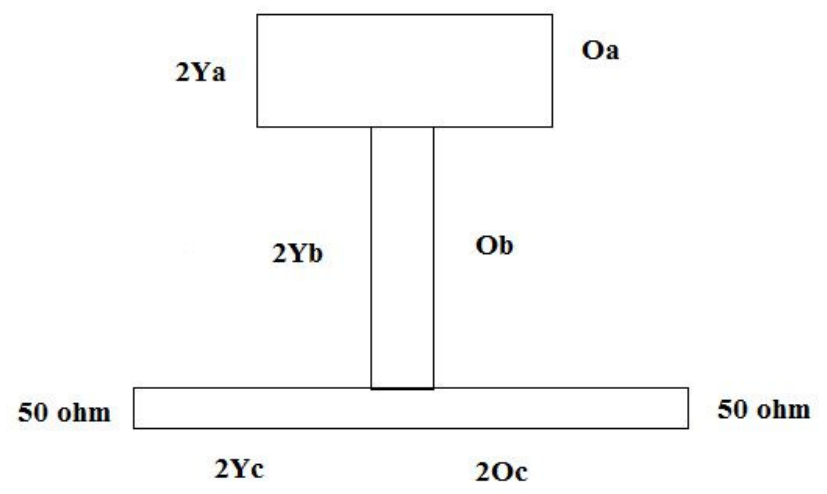

Fig.7 Basic structure of $\mathrm{T}$ shaped resonator with uniform transmission line and stepped impedance stub at the center. 
The actual goal of the simplification is to get the simple expressions of the admittances of $Y_{m}$ and $Y_{n}$ compared with the impedance $Z_{\text {in }}$ and to avoid forcing that their real parts should be equal. So therefore only the imaginary parts of Ym and Yn will compose of the objective function. As the real parts are same we have the expression

$$
\begin{aligned}
& Y_{n}=Y_{m}+j B_{s t u b} \\
& Y_{n}=G_{m}+j B_{m}+j B_{s t u b}
\end{aligned}
$$

The matching at one port means the filter can be matched to any transverse plane of the line that has the characteristic admittance of $Y_{c}$ which divides the filter into two parts. Therefore the matching condition $Y_{n}=Y_{m}^{*}$ leads to

$$
2 B_{m}+j B_{s t u b}=0
$$

Now from the ref [5] the various expressions for the susceptance $B_{m}$ as

$$
\begin{aligned}
B_{m} & =\frac{Y_{c} \tan \theta_{C}\left(1-Y_{p}^{2}\right)}{1+Y_{p}^{2} \tan ^{2} \theta_{C}} \\
B_{\text {stub }} & =2 Y_{b} \frac{k \tan \theta_{a}+\tan \theta_{b}}{1-K \tan \theta_{a} \cdot \tan \theta_{b}}
\end{aligned}
$$

Where $Y_{P}=1 /\left(50 Y_{C}\right)$ and $k=Y_{a} / Y_{b}$. To make sure that the filter gives the Bandpass response the condition of $2 B_{m}+j B_{s t u b}=0$ should be verified for every frequency within the bandwidth of the Bandpass filter. Therefore the new objective function can be formed as

$$
F_{\text {OBJECTIVE }}=\sum_{f N \min }^{f N \max }\left|2 B_{m}+B_{\text {stub }}\right|<\varepsilon \quad \text { (convergence tolerance) }
$$

Which can be simplified by substituting the equations in the objective function

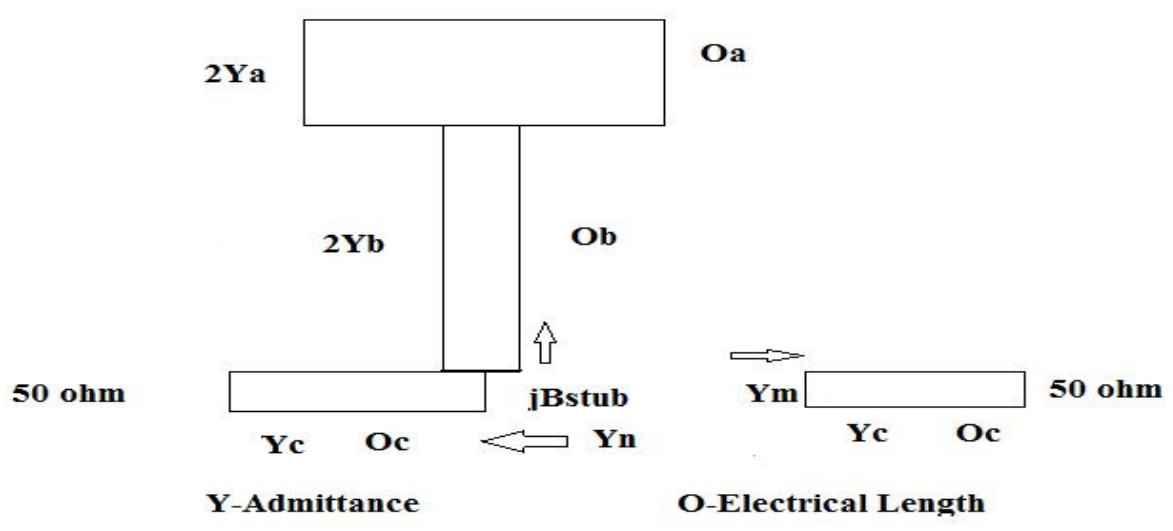

Fig.8 Simplified structure of the filter with their electrical lengths and admittance. 


$$
F_{\text {OBJECTIVE }}=\sum_{f \text { Nmin }}^{f N \max }\left|Y_{c} \frac{Y_{c} \tan \theta_{C}\left(1-Y_{p}^{2}\right)}{Y_{c} \tan \theta_{C}\left(1-Y_{p}^{2}\right)}+Y_{b} \frac{k \tan \theta_{a}+\tan \theta_{b}}{1-K \tan \theta_{a} \cdot \tan \theta_{b}}\right|
$$

Now to have the sharp selectivity at the cutoff frequency at the end of the passband of the filter it is necessary to design the center stub of the filter in such a way that the center stub will place the zeros on the frequency $0.8 \mathrm{GHz}$ and $2.7 \mathrm{GHz}$ which is the cutoff frequency of the filter. That actually means that the filter will have $\left|S_{21}=0\right|$ at the frequency $f_{z 1}=0.8 \mathrm{GHz}$ and $f_{z 2}=$ $2.7 \mathrm{GHz} f_{z 1}$ and $f_{z 2}$ are the two transmission zeros of the center stub also $B_{s t u b}\left(f_{Z 1}\right)=$ $B_{s t u b}\left(f_{Z 2}\right)=\infty$ which could be designed at these frequencies as

$$
k \tan \theta_{a}\left(f_{z i}\right) \cdot \tan \theta_{b}\left(f_{z i}\right)=1 \quad i=(1,2)[8]
$$

Which leads to two expressions

$$
\theta_{02}=\frac{f_{0}}{f_{z i}} \tan ^{-1}\left[\frac{1}{k \tan \left(\theta_{01}\left(\frac{f_{z i}}{f_{0}}\right)\right)}\right] i=(1,2)
$$

To find all possible combinations of $\theta_{02}, \theta_{01}$ and $k$ a numerical iterative method is necessary to verify the expression

$\left|\theta_{02}(2)\right|-\left|\theta_{02}(1)\right|<\varepsilon^{\prime}$ Where $\theta_{01}=0$

The ratio of $\frac{f_{Z 2}}{f_{Z 1}}$ is a function of $\alpha=\frac{\theta_{a}}{\theta_{b+\theta_{a}}}$ for different values of $\mathrm{k}$. The minimum value of $\mathrm{k}$ can be obtained at $\theta_{a}=\theta_{b}=\theta$. So the value of $\alpha=0.5$. In case where $\frac{f_{Z 2}}{f_{z 1}}=3.375$, then

$$
\theta_{x}=\frac{\pi}{\left(\frac{f_{z 2}}{f_{Z 1}}+1\right)}[10] \theta_{y}=\pi-\theta_{x}
$$

$\mathrm{K}$ is less than the maximum value due to characteristic impedance value of the Microstrip line that vary from between $20 \mathrm{ohm}$ to $140 \mathrm{ohm}$. So the K varies from the minimum value to the maximum value of $\mathrm{k}=7$.

\section{DESIGN METHODOLOGY}

This paper uses the structure of the Bandpass filter designed in ref [5].The filter in ref[5] consist of a uniform transmission line with a stepped impedance open stub connected in the center of the line with the three inter-digitized parallel coupled lines at each side of the filter. The filter also uses the defective ground technique to have a better control over the input reflection coefficient of the filter. The filter designed in this paper is designed for the frequency range of $0.8 \mathrm{GHz}$ to $2.7 \mathrm{GHz}$ but the filter designed in ref[5] is designed for 3.1 to $10.6 \mathrm{GHz}$. The filter is designed in this paper uses the FR4 substrate having the dielectric constant of er $=4.6$ the substrate height of $\mathrm{h}=1.6 \mathrm{~mm}$ and conductor thickness of $\mathrm{T}=0.01 \mathrm{~mm}$. 
International Journal Of Microwave Engineering (JMICRO) Vol.1, No.3, July 2016

\begin{tabular}{|c|c|c|}
\hline Reference & $\begin{array}{c}\text { Dielectric/substrate } \\
\text { Height(mm) }\end{array}$ & SF \\
\hline$[1]$ & $10.8 / 1.27$ & 0.642 \\
\hline$[2]$ & $10.8 / 1.27$ & 0.594 \\
\hline$[3]$ & $2.55 / 0.8$ & 0.926 \\
\hline$[4]$ & $2.55 / 0.8$ & 0.921 \\
\hline$[5]$ & $2.33 / 0.5$ & 0.861 \\
\hline This paper & $4.6 / 1.6$ & 0.9032 \\
\hline
\end{tabular}

Table 1. Comparison of the filter structures with their substrate and selectivity factor.

The filter is then integrated with the Low noise amplifier from Analog Devices ADL5544. The amplifier has a good gain response in the frequency range of $0.1 \mathrm{GHz}$ to $6 \mathrm{GHz}$. The amplifier is made stable in the frequency range by proper biasing the Amplifier by the biasing circuit. The filter and the amplifier circuit is separately designed and then integrated. The filter has a good response in the passband of the filter and sharp selectivity after the upper cutoff frequency. The selectivity factor of the filter is 0.9032 as the frequency at $-3 \mathrm{~dB}$ is obtained as $2.8 \mathrm{GHz}$ and frequency at $-30 \mathrm{~dB}$ is $3.100 \mathrm{GHz}$. The filter size is about $7 \mathrm{~cm}$ in length and $4 \mathrm{~cm}$ in height. This configuration of the filter is chosen as the filter has to be fabricated in India. The FR4 material of er=4.6 is easily available for fabrication. The minimum spacing between the tracks which can be fabricated in India is $0.2 \mathrm{~mm}$. Hence no length or spacing between the tracks is not less than 0.2 $\mathrm{mm}$. The layout of the filter is drawn using ADS layout window and momentum simulation is done to obtain the frequency response of the designed filter.

As shown in Fig. 8 the filter consist of center stub and a uniform impedance line of $\lambda / 4$ at the center frequency of $1.75 \mathrm{GHz}$. The center stub is connected in the center of the uniform impedance line and the lengths and the widths of center stubs are taken such that the zeros are placed at the cutoff frequencies of the filter. The inter-digital coupling is made on both side of the filter to suppress the frequencies after the cutoff frequency and to have a larger stopband.

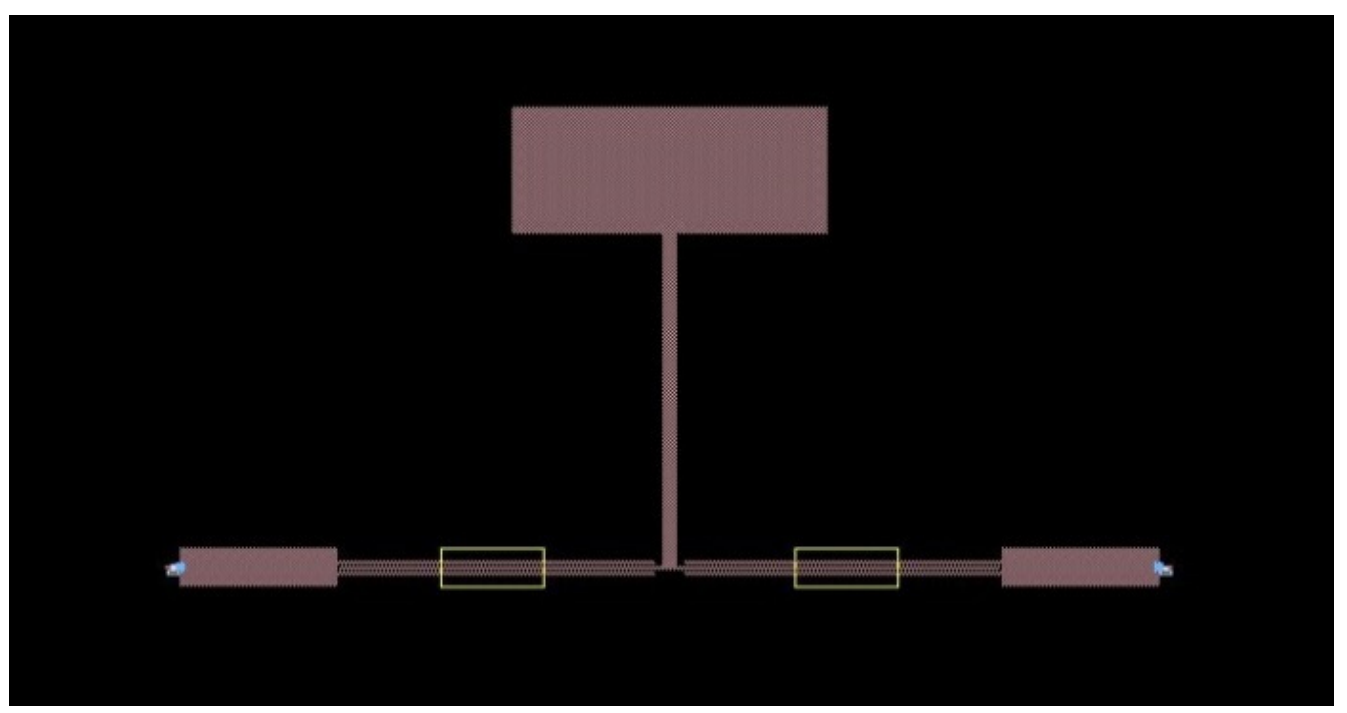

Fig.9 The layout of wideband Bandpass filter. 
The filter also uses the defective ground structure in which the ground plane is not present below the coupling. The lengths of the defective ground can be obtained by the trial and error method. The defective ground structure minimizes the input return loss of the filter. The filter gain response shows that the filter has the passband from $0.8 \mathrm{GHz}$ to $2.7 \mathrm{GHz}$. The filter has an insertion loss of about $0.5 \mathrm{~dB}$.

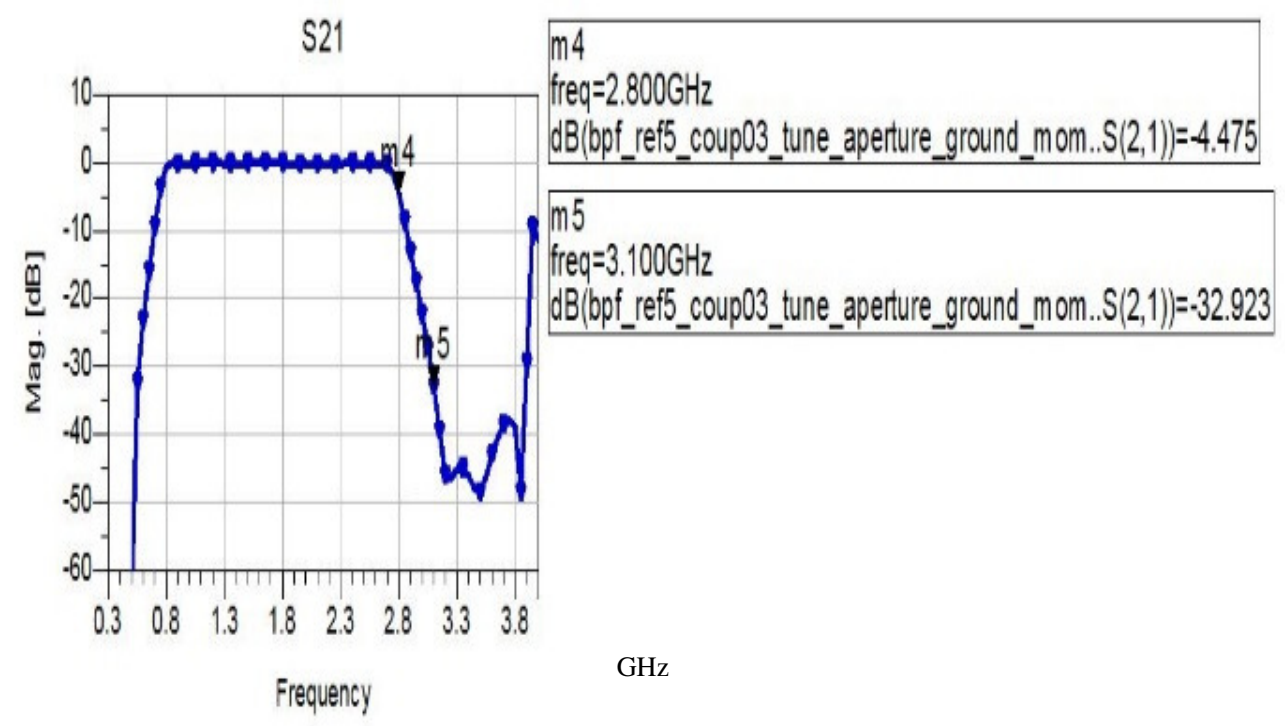

Fig.10 The output gain (S21) of wideband Bandpass filter.

The Fig.9 and fig.10shows the momentum simulation results of the filter. The filter shows its passband from $0.8 \mathrm{GHz}$ to $2.7 \mathrm{GHz}$ the $\mathrm{S} 11$ as shown in Fig. 10 is below $-10 \mathrm{~dB}$ for the whole passband of concern. The filter suffers from the second harmonics of the filter center frequency.

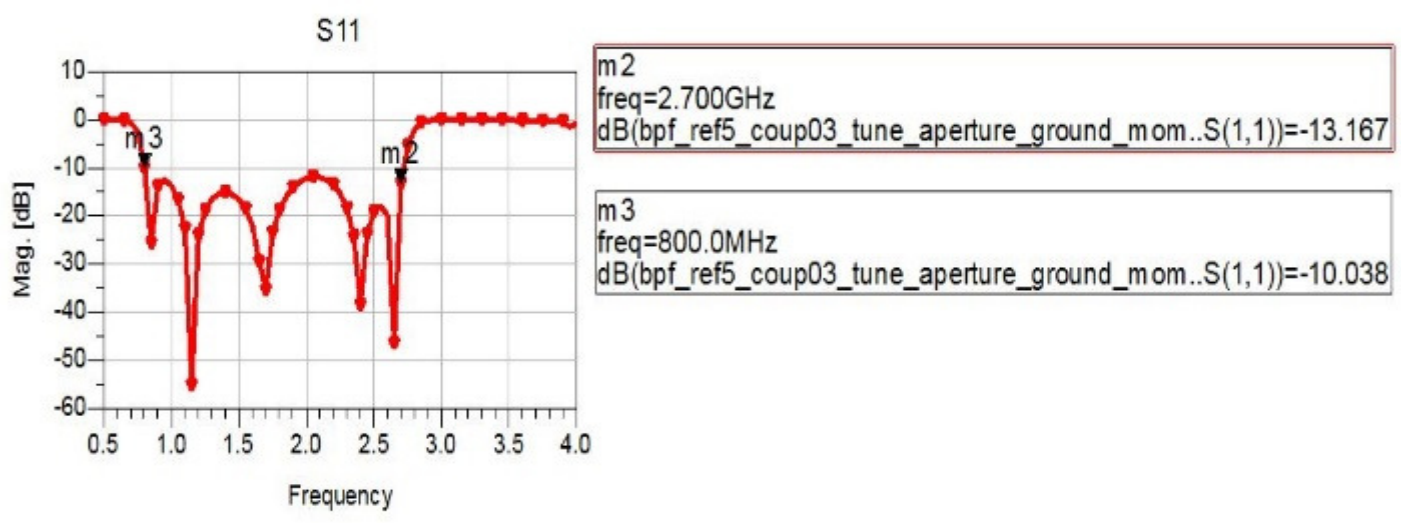

$\mathrm{GHz}$

Fig.11 The input return loss (S11) of wideband Bandpass filter. 
The stopband of the filter is extended up-to $4 \mathrm{GHz}$. The designed filter uses the compact structure proposed in ref [5] hence is compact in size for the proposed frequencies of $0.8 \mathrm{GHz}$ to $2.7 \mathrm{GHz}$. The size of the filter is only $7 \mathrm{~cm}$. As the filter is designed for the low frequencies as compared to ref [5] the lengths of the filter are greater. The filter is designed to cover all the wireless applications which work in $800 \mathrm{MHz}$ to $2.7 \mathrm{GHz}$ band of frequencies

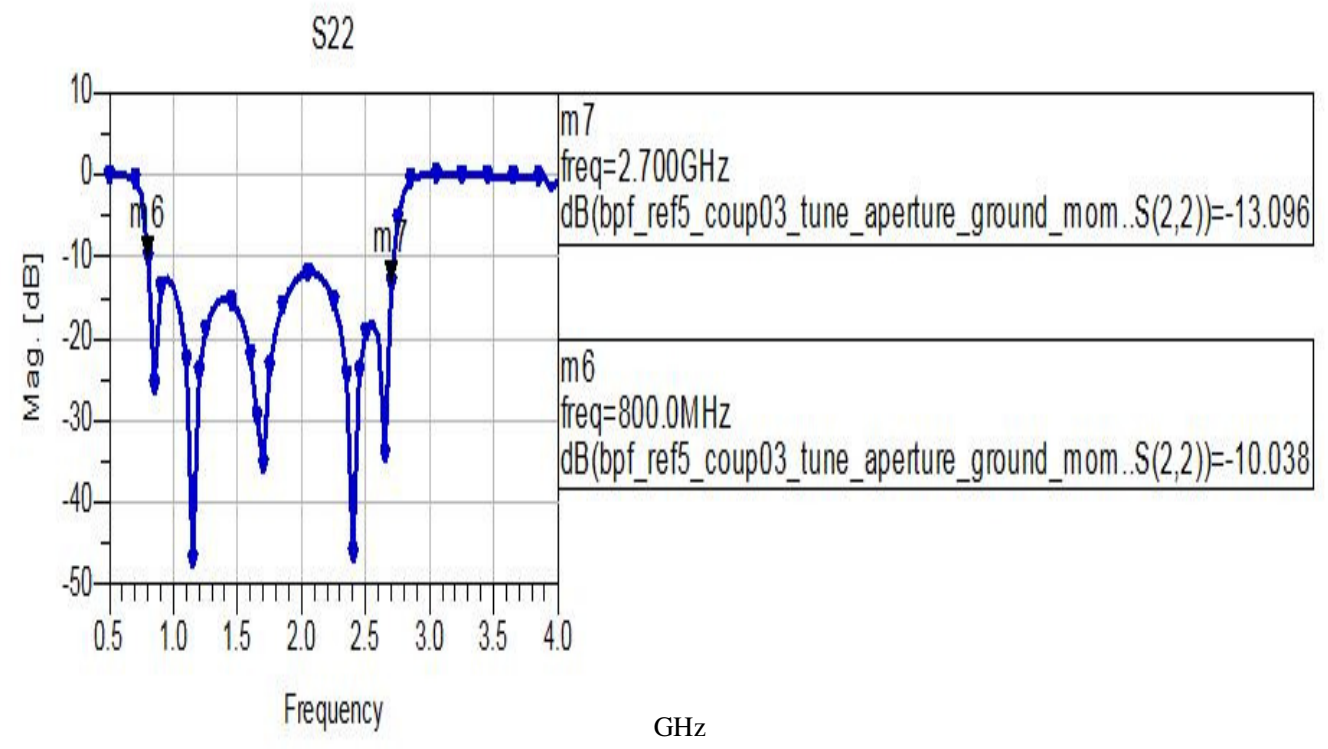

Fig.12 The output return loss (S22) of wideband Bandpass filter

The output return loss of the simulated filter as shown in Fig. 11 is less than $-10 \mathrm{~dB}$ for the whole passband of concern. The filter has the $\mathrm{S} 22$ of $-10.038 \mathrm{~dB}$ at $800 \mathrm{MHz}$ and $-13.096 \mathrm{~dB}$ at $2.7 \mathrm{GHz}$ and output return loss remains below $-10 \mathrm{~dB}$ between this band of frequency. The simulation result of output isolation of the filter is shown in Fig.12.

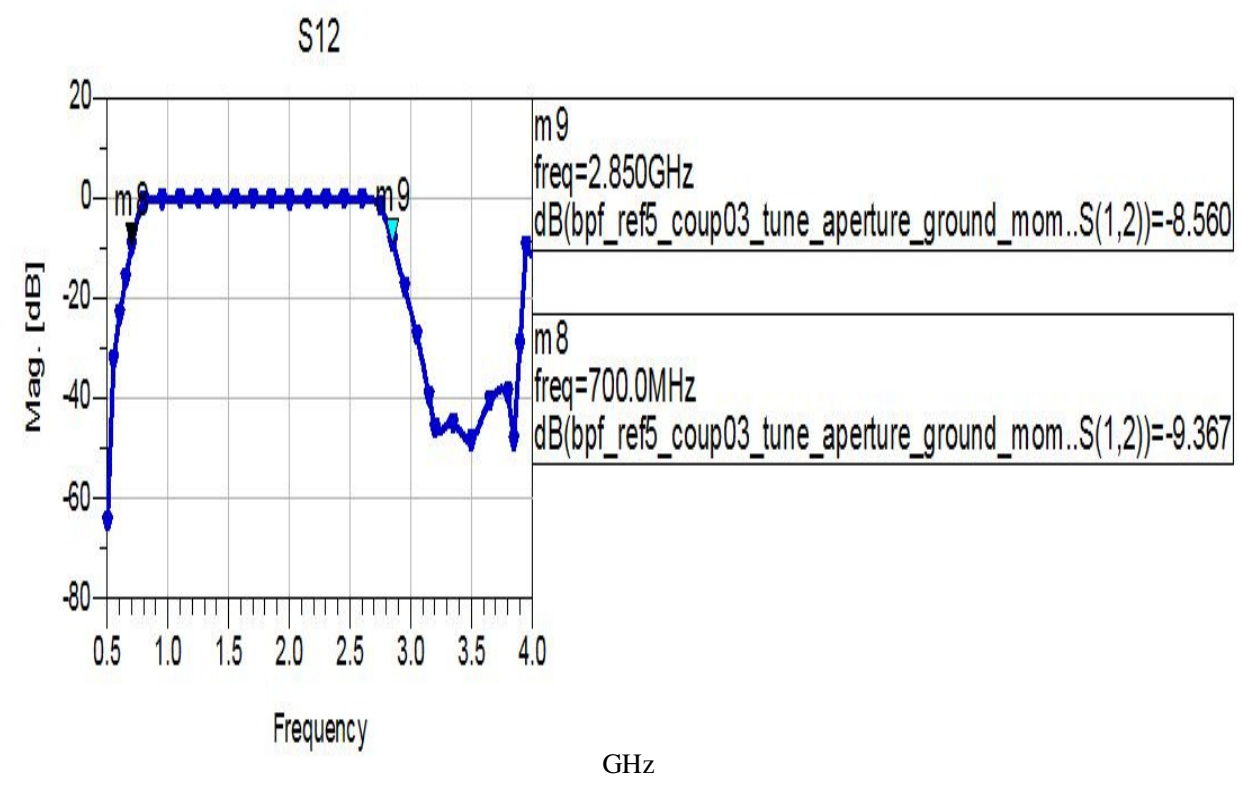

Fig.13 The output isolation (S12) of wideband Bandpass filter. 
The output isolation of the filter is $-9.367 \mathrm{~dB}$ at $700 \mathrm{MHz}$ and $-8.560 \mathrm{~dB}$ at $2.85 \mathrm{GHz}$. The filter has the good isolation in the stopband of the filter.

\section{FILTER INTEGRATED WITH LNA}

The filter designed in the section III is integrated with a low noise amplifier to further increase the gain of the signal filtered by the Bandpass filter. The low noise amplifier actually amplifies the signal obtained from the Bandpass filter and adds its self-submicron noise as minimum as possible. Low noise amplifier adds minimum noise to the circuit but amplifies the signal with the expected gain. Hence it is called as low noise amplifier. The low noise amplifier used in the design is from the analog devices ADL5544 which has good gain of $15 \mathrm{~dB}$ in the range of 300 $\mathrm{MHz}$ to $2.7 \mathrm{GHz}$. The amplifier is made stable in $0.8 \mathrm{GHz}$ to $2.7 \mathrm{GHz}$ by designingthe biasing circuit of the amplifier. The biasing circuit of the amplifier can also be found in the data sheet of the amplifier. The stability of the amplifier is checked through Rollet's criteria and Stab fact function in ADS schematic.

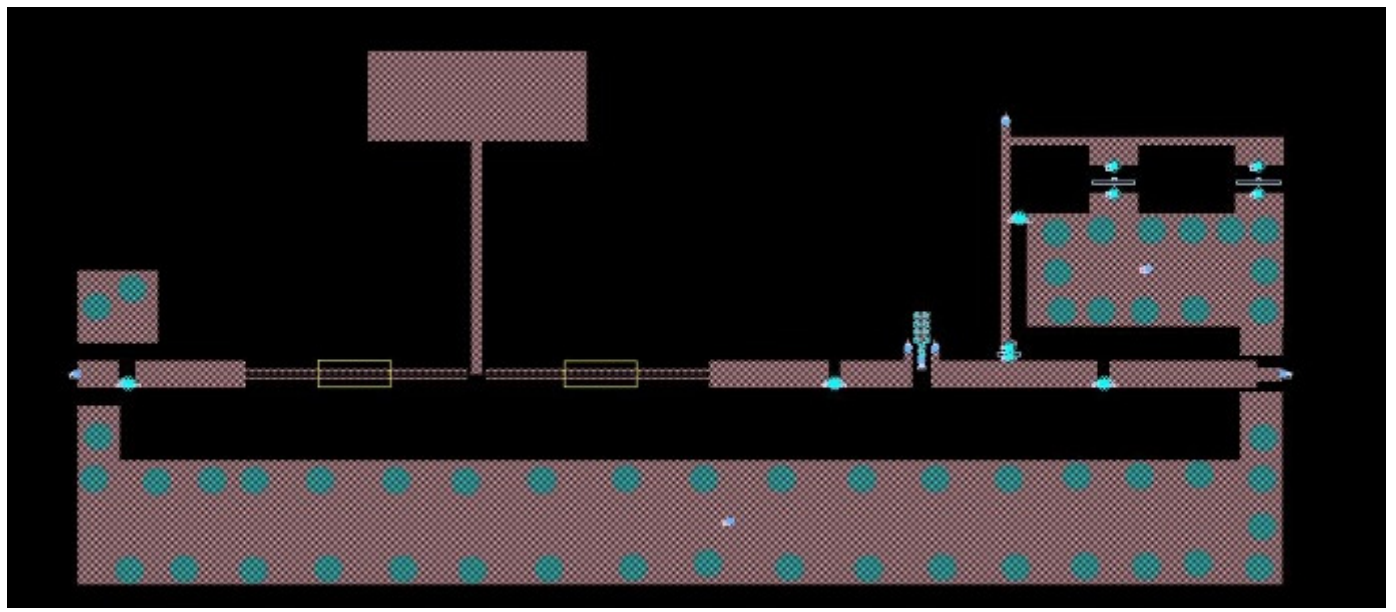

Fig.14 The layout of the filter integrated with LNA and the basing circuit of amplifier.

Figure 13 shows the integrated layout of the filter and the amplifier various different components for amplifier biasing are used like choking inductors and decoupling capacitors. The component padding and IC padding of the amplifier can be shown in the Fig.13. The IC padding in the layout is taken from the datasheet of the IC used. The inductors and the capacitor padding is taken according to the package size of the component e.g. 0603 or 5750. Now in comparison the 0603 package is small in size as compared to that of the 5750 package. The inductors and the capacitors are used from the Murata and Coilcraft. The small circles shown in the Fig.13 are Via which connects the upper or the top metal layer to the lower ground plane. The ground plane should be as large as possible in RF/Microwave circuits to minimize the radiation losses of the circuit otherwise the losses of the circuit increase and the signal travelling through Microstrip gets attenuated earlier due to excessive losses of radiation. The design of the filter is maintained to have minimum Microstrip losses.

\section{FILTER HARDWARE RESULTS}

The hardware results are tested on the VNA (Vector network analyzer) which can test the circuit up to $8 \mathrm{GHz}$. The dc voltage source is required for the amplifier biasing. The dc voltage source of $3 \mathrm{~V}$ is used for the amplifier ADL5544. 
International Journal Of Microwave Engineering (JMICRO) Vol.1, No.3, July 2016

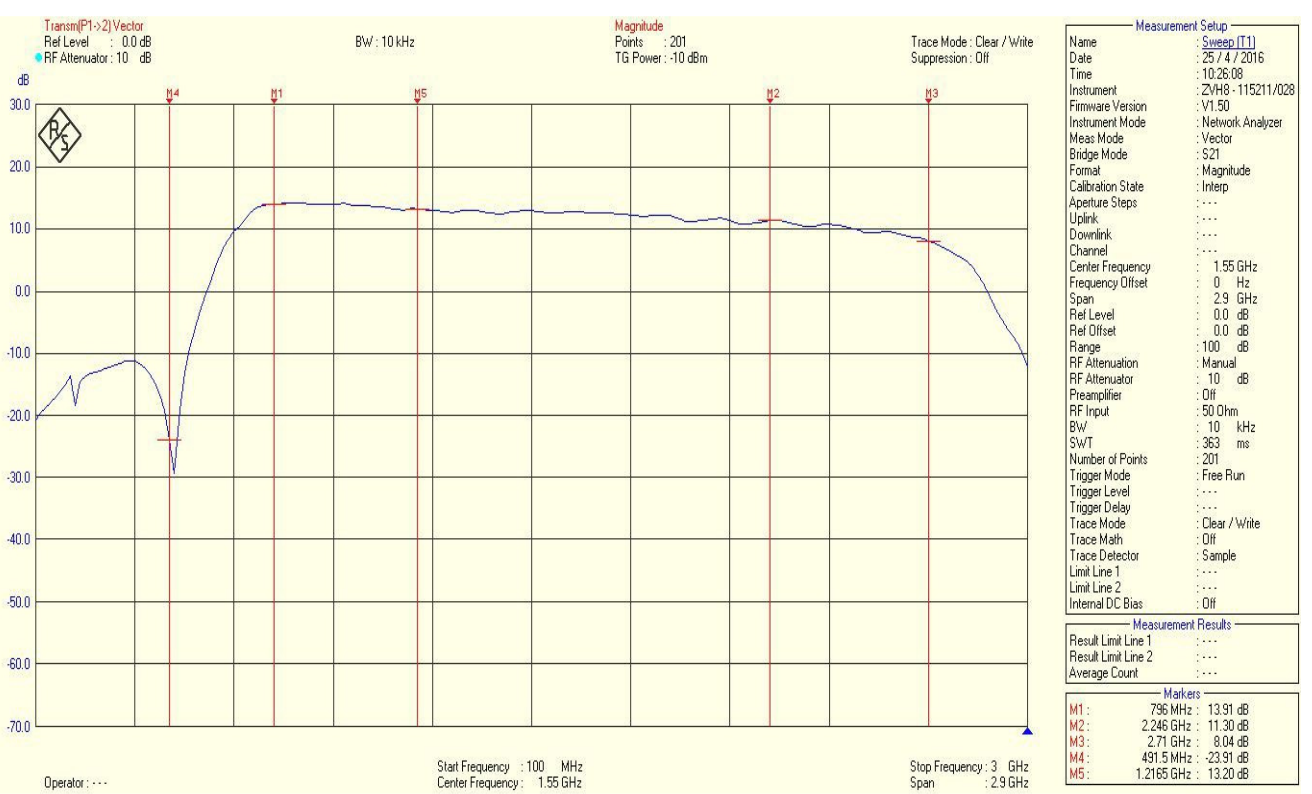

Fig.15 The output gain (S21) of the wideband Bandpass filter with integrated low noise amplifier tested on VNA.

Figure 14 shows the measured gain S21 of the designed filter integrated with amplifier. It is observed that the design has the gain of $-30 \mathrm{~dB}$ at $500 \mathrm{MHz}$ then $13.91 \mathrm{~dB}$ at $791 \mathrm{MHz}$ then $13.20 \mathrm{~dB}$ at $1.2165 \mathrm{GHz}$ then $11.30 \mathrm{~dB}$ at $2.246 \mathrm{GHz}$ and $8.04 \mathrm{~dB}$ at $2.71 \mathrm{GHz}$. The design has the gain roll-off of $2 \mathrm{~dB}$ in the passband of the filter.

Figure 15 shows the gain over the whole frequency range of $8 \mathrm{GHz}$. The filter shows the sharp selectivity at cutoff frequencies of the filter. The filter has a good sharp attenuation in the stopband of the filter. The filter is affected with the second harmonics of the center frequency of the filter. Hence the gain plot rises after $4.5 \mathrm{GHz}$.
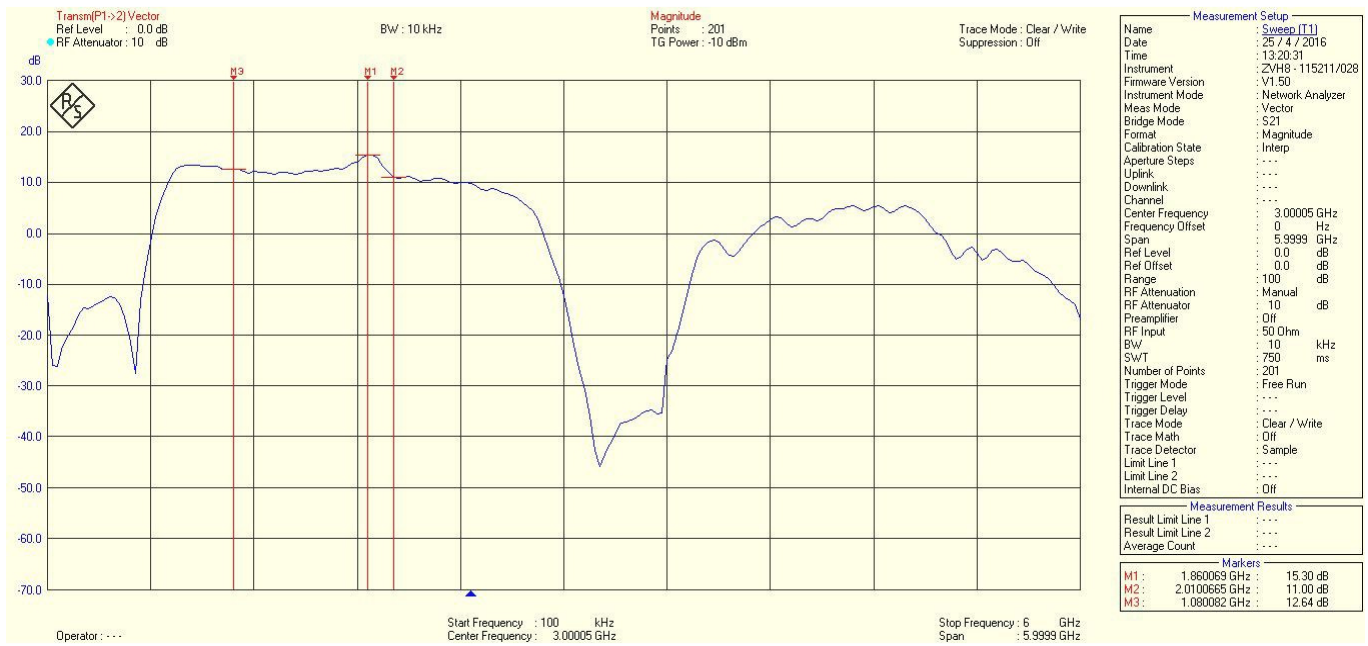

Fig.16 The output gain (S21) of wideband Bandpass filter with integrated low noise amplifier tested on VNA for full frequency range of $8 \mathrm{GHz}$ 
The Microstrip filters are all affected with the harmonics this is the disadvantage of Microstrip filter to have smaller Stopbands after the cutoff frequency. The input return loss of the measured filter as shown in Fig.16 is less than $-8 \mathrm{~dB}$.

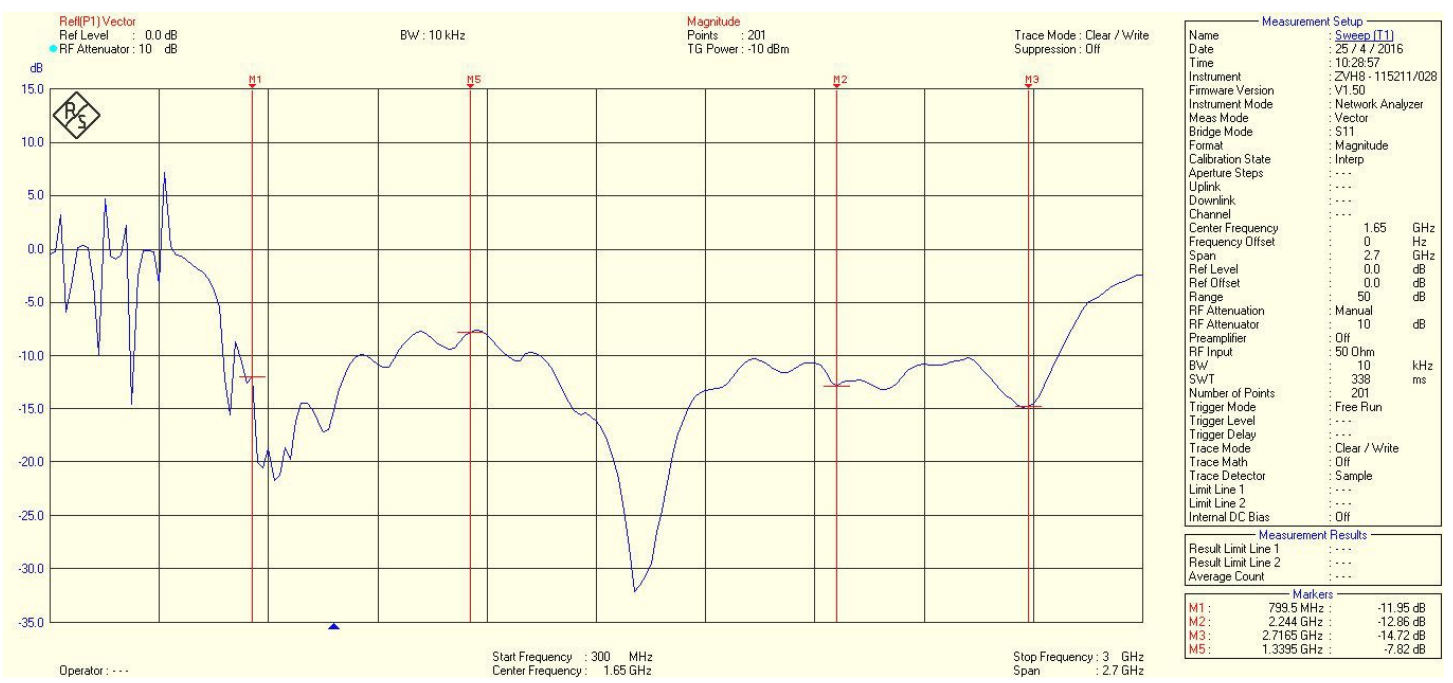

Fig.17 The input return loss (S11) of the measured wideband Bandpass filter integrated with amplifier.

The filter measures the input return loss $\mathrm{S} 11$ of $-11.8 \mathrm{~dB}$ at $800 \mathrm{MHz}, 7.82 \mathrm{~dB}$ at $1.335 \mathrm{GHz}$, $12.86 \mathrm{~dB}$ at $2.244 \mathrm{GHz}$ and $-14.72 \mathrm{~dB}$ at $2.7165 \mathrm{GHz}$. For rest of the frequencies the input return loss stays below $-10 \mathrm{~dB}$.The measured result of the filter are satisfactory as compared to the simulation result $\mathrm{S} 11$ of the filter.

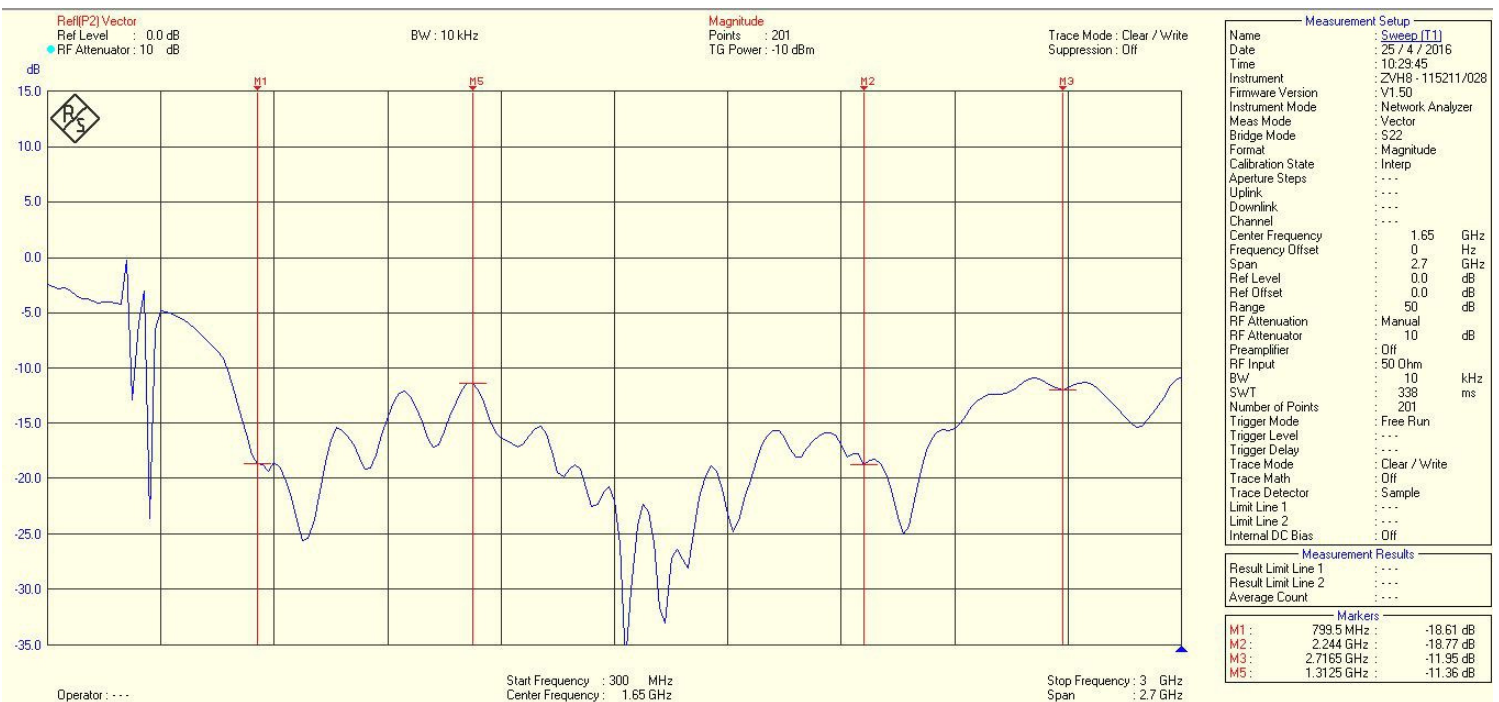

Fig.18 The output return loss (S22) of the measured wideband Bandpass filter integrated with amplifier.

Figure 17 shows the measured result of output return loss S22. The output return loss is less than $-10 \mathrm{~dB}$ for the whole passband of frequencies. The output return loss is measured as $-18.61 \mathrm{~dB}$ at $800 \mathrm{MHz},-11.36 \mathrm{~dB}$ at $1.3125 \mathrm{GHz},-18.77 \mathrm{~dB}$ at $2.244 \mathrm{GHz}$ and $-11.95 \mathrm{~dB}$ at $2.7165 \mathrm{GHz}$. 

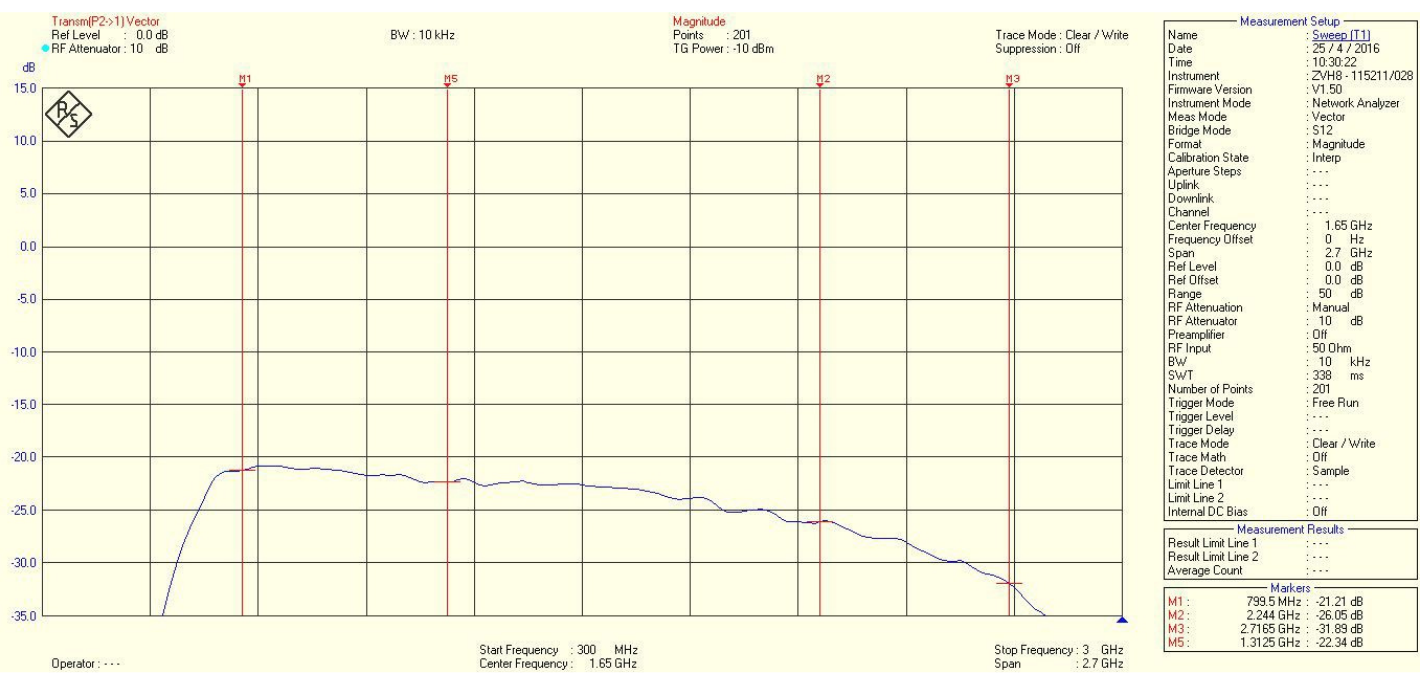

Fig.19 The output isolation (S12) of the measured wideband Bandpass filter integrated with amplifier.

The simulation and measured result of S22 are in good agreement with each other. Figure 18 shows the output isolation of the filter. The output isolation of the filter is below $-20 \mathrm{~dB}$ for the whole frequency range of operation. The measured $\mathrm{S} 12$ has $-21.21 \mathrm{~dB}$ at $800 \mathrm{MHz},-22.34 \mathrm{~dB}$ at $1.3125 \mathrm{GHz},-26.05 \mathrm{~dB}$ at $2.244 \mathrm{GHz}$ and $-31.89 \mathrm{~dB}$ at $2.7165 \mathrm{GHz}$. The measured and simulated results of S12 are satisfactory when compared to each other.

\section{FABRICATION AND TESTING}

As the design was to be fabricated in India the minimum spacing between the two tracks was not less than $0.2 \mathrm{~mm}$. So the results were obtained keeping the minimum distance between tracks as $0.21 \mathrm{~mm}$.

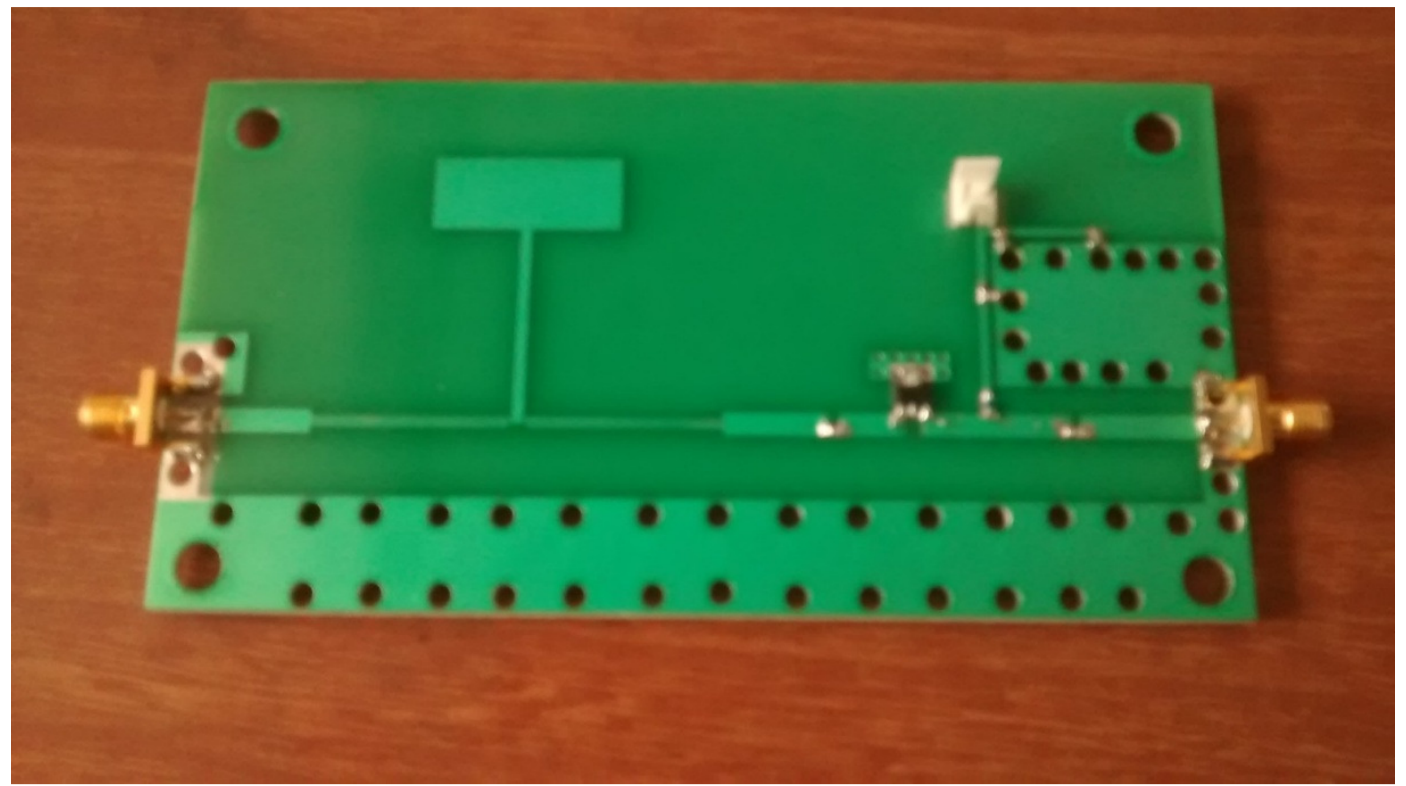

Fig.20 Fabricated PCB of the filter and LNA with assembled components and SMA connectors. 
Figure 19 shows the PCB of the filter and amplifier IC assembled to the PCB with other components. The SMA connectors are connected to the input and the output ports so that the PCB can be tested on VNA (Vector network analyzer). The 2 pin connecter is connected to supply the dc voltage to the amplifier IC. The filter is designed using the defective ground at the back of the inter-digital coupled lines from both the sides. Fig.20 shows the defective ground of the fabricated filter. The defective ground helps the designer to vary the coupling between the lines as per required. The lengths and the widths of the defective ground are taken by trial and error method

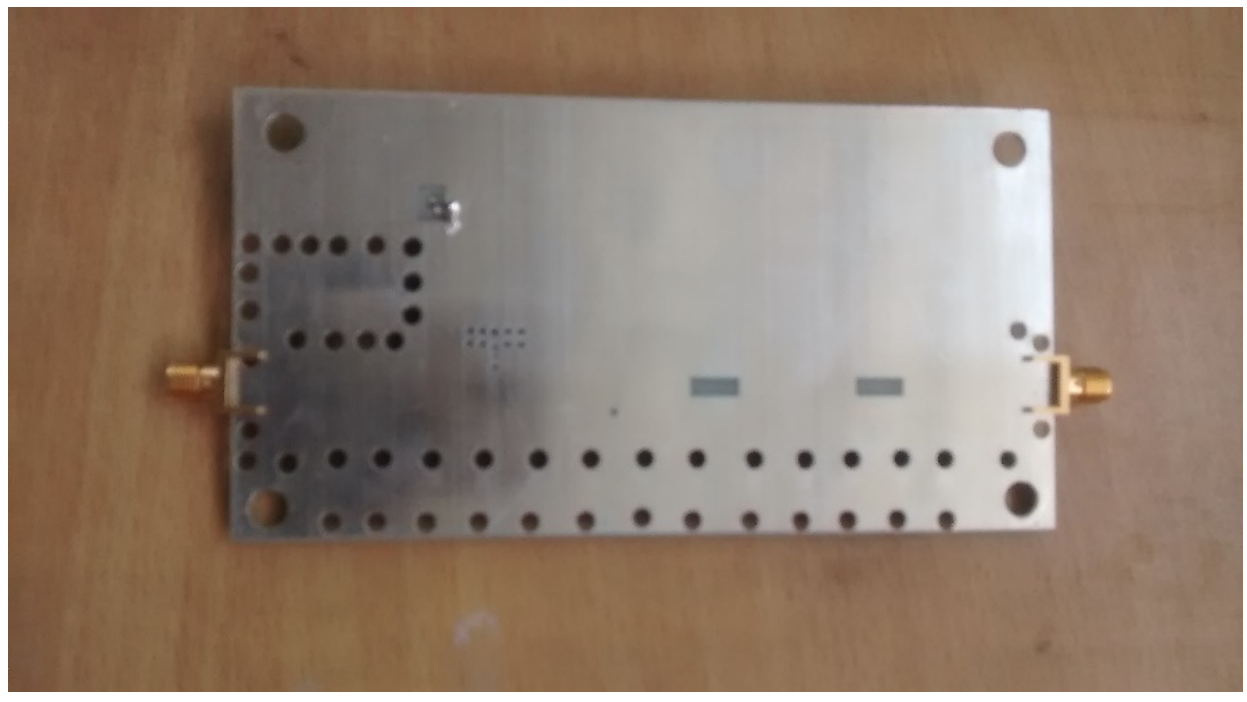

Fig.21 Backside of the fabricated PCB of the filter which shows the defective ground.

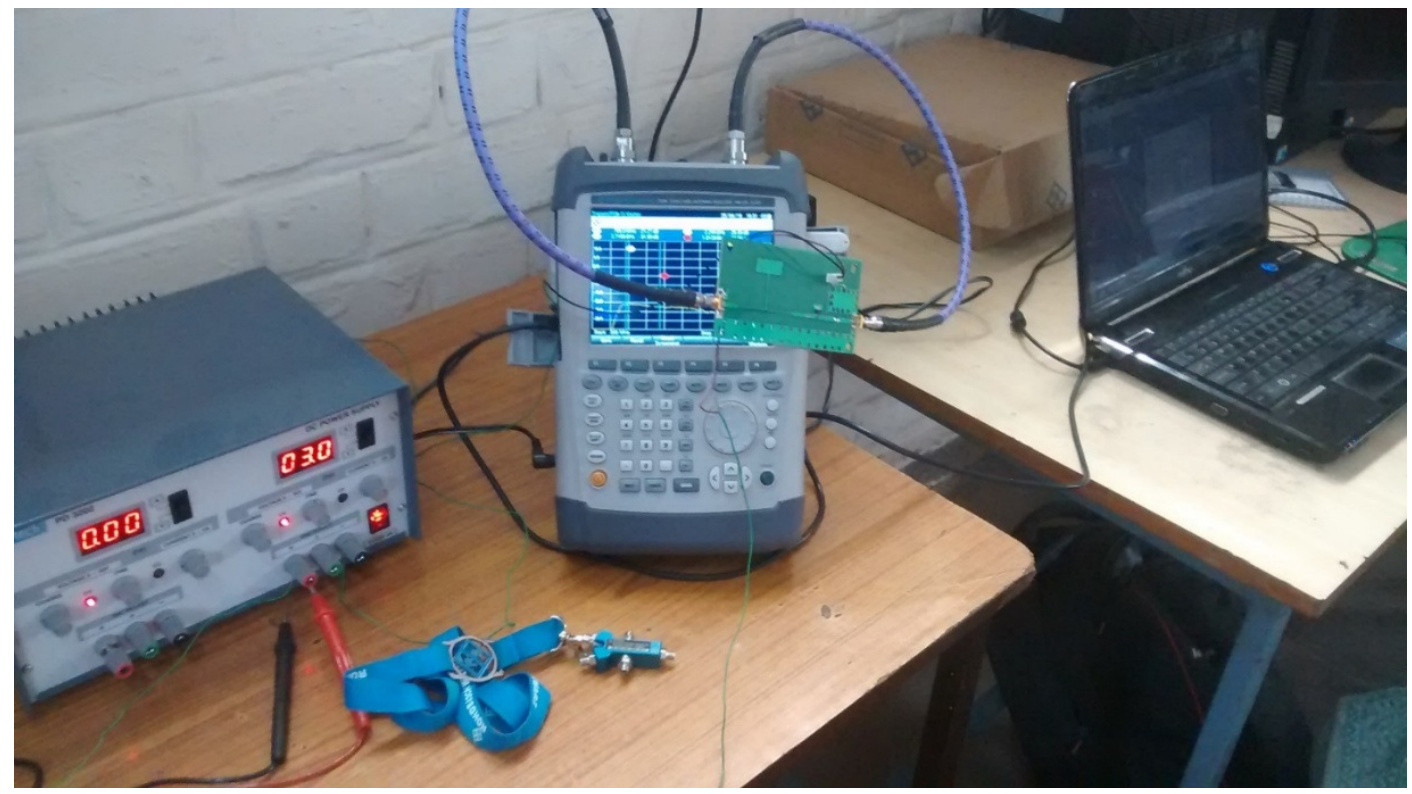

Fig.22 The testing setup for measured results of the filter which shows the VNA and dc power supply connected to the fabricated PCB. 
International Journal Of Microwave Engineering (JMICRO) Vol.1, No.3, July 2016

The defective ground concept helps to improve the input/output return loss of the filter. Varying the length and the width too much can change the passband of the filter completely. The defective ground actually does not have a ground plane in that patch of the PCB due to which the coupling degree of the filter can be changed and varied. The length of the defective ground taken in this design is length $=8 \mathrm{~mm}$ and width $=2.97 \mathrm{~mm}$. This lengths and widths are found out by trial and error methods which can vary as per the frequency of operation. Fig.21 shows the testing setup for the fabricated filter. Testing was done on VNA from Rohde \& Schwarz model no ZVH8 which can measure from $100 \mathrm{KHz}$ to $8 \mathrm{GHz}$. The VNA is first calibered correctly to open, shorts and $50 \mathrm{ohm}$ terminations so that the testing results are obtained with accordance to the calibrated references.

\section{CONCLUSION}

The study has revealed that the design developed in the ref [5] is the best design in terms of the performance and size of the filter compared to the various other designs developed earlier. The filter is designed to operate at frequencies of $0.8 \mathrm{GHz}$ to $2.7 \mathrm{GHz}$. The filter has the good performance in the pass band as well as in stopband. The input return loss of the simulated results of the filter is less than $-10 \mathrm{~dB}$ in the entire passband and that in hardware result is less than $-7 \mathrm{~dB}$. The filter suffers the maximum of only $0.5 \mathrm{~dB}$ of insertion loss.The gain $\mathrm{S} 21$ of the proposed design is constant in whole passband of having a gain roll-off of $2 \mathrm{~dB}$ in the passband of the filter. The output return loss S22 of simulated and the measured results is less than $-10 \mathrm{~dB}$. The output isolation S12 of the design is less than $-20 \mathrm{~dB}$.The selectivity factor of the proposed filter is about 0.9032 which is greater than the filter of ref [5]. The proposed filter is designed for the lower frequency then that of the ref [5] so the size of the proposed filter is greater than that of the filter designed in ref [5] as all the lengths of the filter depends on the wavelength of the center frequency of the band of operation of the filter. As the center frequency is much lower the wavelength is higher. Hence the lengths are higher. The filter uses the substrate of FR4 with the dielectric of er $=4.6$ and height $=1.6 \mathrm{~mm}$ and metal thickness of $t=0.01 \mathrm{~mm}$. The filter has the sharper roll off with compact size as compared to the other filters in this frequency range. All the simulations are carried out by ADS software momentum simulation and the fabricated hardware is tested using the VNA (Vector network analyzer) and is observed that the hardware results are in good comparison with that of the simulated results.

\section{ACKNOWLEDGEMENTS}

The authors wish to thank Prof. R.P. Patil for her valuable guidance and RFIC solutions for their support and suggestions that they have given for the completion of this work.

\section{REFERENCES}

[1] L. Zhu, S. Sun, and W. Menzel, "Ultra-wideband (UWB) Bandpass filters using multiple-mode resonator," IEEE Microwave. Wireless Component, Letter, vol. 15, no. 11, pp. 796-798, Nov. 2005.

[2] R. Li and L. Zhu, "Compact UWB Bandpass filter using stub-loaded multiple-mode resonator," IEEE Microwave. Wireless Component. Letter, vol.17, no. 1, pp. 40-42, Jan. 2007.

[3] Q.-X. Chu and X.-K. Tian, "Design of UWB Bandpass filter using stepped-impedance stub-loaded resonator," IEEE Microwave. Wireless, Component. Letter, vol. 20, no. 9, pp. 501-503, Sep. 2010.

[4] Q.-X. Chu, X.-H. Wu, and X.-K. Tian, "Novel UWB Bandpass filter Using stub-loaded multiple-mode resonator," IEEE Microwave. Wireless Component. Letter, vol. 21, no. 8, pp. 403-405, Aug. 2011. 
[5] AbdelkaderTaibi, Mohamed Trabelsi, AbdelhalimSlimane, MohandTaharBelaroussi, Member, IEEE, and Jean-Pierre Raskin, Fellow, IEEE"A Novel Design Method for Compact UWB Bandpass Filters" IEEE microwave and wireless component letters .2014.

[6] Q. X. Chu and S. T. Li, "Compact UWB Bandpass filter with improvedUpper-stopband performance," Electron Letter., vol. 44, no. 12, pp.742-743, Jun. 2008.

[7] B. Y. Yao, Y. G. Zhou, Q. S. Cao, and Y. C. Chen, "Compact UWBBandpass filter with improved upper-stopband performance," IEEE Microwave. Wireless Component. Letter, vol. 19, no. 1, pp. 2729, Jan. 2009.

[8] L. Zhu and W. Menzel, "Compact Microtrip Bandpass filter with two transmission zeros using a stubtapped half-wavelength line resonator,” IEEE Microwave. Wireless Component. Letter, vol. 10, no. 1, pp. 16-18, Jan. 2003.

[9] L. Zhu, H. Bu, and K. Wu, "Aperture compensation technique for innovative design of ultra-broadband Microstrip Bandpass filter,” in IEEE MTT-S Int. Dig., Jun. 2000, vol. 1, pp. 315-318, vol. 1.

[10] L. Zhu, H. Bu, K. Wu, and M. S. Leong, "Miniaturized multi-pole broad-band Microstrip Bandpass filter: concept and verification," in Proc. 30th Eur. Microwave. Conf., Paris, France, Oct. 2000, vol. 3, pp.334-337.

[11] L. Zhu, H. Bu, and K. Wu, "Broadband and compact multi-pole Microstrip Bandpass filters using ground plane aperture technique," Proc. Inst. Elect. Eng., vol. 147, no. 1, pp. 71-77, 2002.

[12] S. W. Wong and L. Zhu, "Quadruple-mode UWB Bandpass filter with improved out-of-band rejection," IEEE Microwave. Wireless Component. Letter, vol. 19, no. 3, pp. 152-154, Mar. 2009.

[13] H. W. Deng, Y. J. Zhao, L. Zhang, X. S. Zhang, and S. P. Gao "Compact quintuple-mode stub-loaded resonator and UWB filter,” IEEE Microwave. Wireless Component. Letter, vol. 20, no. 8, pp. 438-440, Aug. 2010.

[14] M. Z. Ji and Q. X. Chu, “Compact UWB Bandpass filter using pseudo Inter-digital stepped-impedance resonators," in Proc. China Micro. Millimeter-Wave Conf., Ningbo, China, Oct. 2007, pp. 1096-1098.

[15] J.-S. Hong and M. J. Lancaster, "Chapter 10 advanced RF/microwave filters," in Microwave Filters for RF/Microwave Applications. New York: Wiley, 2001

[16] Y. Feng and S. Huang, "The Analysis of Sinusoidal Modulated Method Used for Measuring Fluorescence Lifetime” Spectroscopy and Spectral Analysis. vol. 27, no. 12, pp. 2523-2526, 2007 